\title{
Ultra-High-Performance Concrete Shear Keys in Concrete Bridge Superstructures
}

\author{
Elsy Y. Flores, Jordan Varbel, Craig M. Newtson*, and Brad D. Weldon \\ Department of Civil Engineering, New Mexico State University, Las Cruces, NM 88003
}

\begin{abstract}
Many existing bridges have adjacent girders that utilize grouted shear keys to transfer loads laterally across the superstructure. Cracking and leaking often cause degradation of the shear key and the girder. This work investigates the potential for using non-proprietary ultra-high performance concrete (UHPC) as a grouting material for repair of deteriorated shear keys by testing bond strength between UHPC and substrate concrete surfaces that were either formed or scarified by chipping. Bond strengths were adequate for both surface textures even though texture depth was substantially less than recommended by ACI 546. Scanning electron microscopy has also been used to investigate the bonded area. This microscopic scanning has shown fly ash residue remaining on the substrate after bond failure, indicating that the supplementary cementitious materials produce much of the bond. Ongoing work for this project also includes full-scale testing of UHPC shear keys between pre-stressed channel girders.
\end{abstract}

\section{Introduction}

Precast girders are commonly used in North America due to their ease of construction, affordability, favorable aesthetic appeal, and high flexural stiffness. Some girder shapes require shear keys to ensure monolithic behavior and load transfer across the superstructure. However, shear key durability is a concern to engineers since shear key degradation can jeopardize the integrity of the structure. Transportation agencies have reported that $75 \%$ of distress in adjacent girder bridges was due to cracking along shear keys and de-bonding of the interfaces between the girders and the shear keys [1].

The work presented in this paper investigates the potential for using locally produced ultra-high performance concrete (UHPC) as a grouting material to rehabilitate shear keys in adjacent prestressed girder bridges in New Mexico, USA. Previous research at New Mexico State University has shown that locally produced UHPC has excellent mechanical and durability properties [2]. UHPC has also been shown to have good bonding characteristics $[3,4]$ that are desirable in a potential grouting material. Additionally, the use of nonproprietary UHPC mixtures provides an economical and sustainable alternative to proprietary products. In comparison to other proprietary mixtures, locally produced UHPC has a decrease in cost of anywhere from 10 to $70 \%$ [5]. To evaluate UHPC for potential use as a grout in shear keys, bond between UHPC and substrate concrete was studied. Ongoing work also includes shrinkage testing and full-scale testing of UHPC grouted shear keys.

\section{Background}

\subsection{UHPC}

UHPC typically consists of cement, ground quartz, fine sand, an accelerating admixture, a high-range water reducing admixture (HRWRA), and approximately 2\% by volume of steel fibers [1]. According to ASTM C1856, UHPC has a minimum compressive strength of $120 \mathrm{MPa}(17,000 \mathrm{psi})$. Additionally, UHPC has low porosity, high density, and excellent durability and resistance to chemical and physical attack [6-9]. Another important characteristic of UHPC is that it exhibits better bond to different surface roughnesses compared to normal grout materials [1].

\subsection{Shear keys}

Shear keys are primary structural components of a bridge superstructure. They are located between adjacent girders and are designed to restrain lateral displacement and facilitate load transfer between the girders. Shear key degradation may include debonding cracking and leaking that can compromise the strength and serviceability of the bridge. As a result, individual beams may have to carry entire wheel loads. As damage progresses, differential displacements between girders can cause cracking in overlays and in the shear keys [10].

Several geometric configurations have been studied to improve shear key performance. Some configurations include the design of a shear key at partial, medium, and full depth. Grouting materials, such as non-shrink grout,

\footnotetext{
* Corresponding author: newtson@,nmsu.edu
} 
epoxy grout, engineered cementitious composites, and UHPC have also been investigated [11].

Transverse post-tensioning improves load transfer across shear keys by creating a more monolithic behavior [10]. Post-tensioning also protects the bond between the shear key grout and a girder by keeping the bonded interface compressed. Other studies have shown that the post-tensioning force required to ensure monolithic behavior is dependent on the width of the bridge and the depth of the girder $[12,13]$.

\section{Methodology}

\subsection{UHPC and substrate mixtures}

Proportions for the UHPC and substrate concrete mixtures are presented in Tables 1 and 2, respectively. The UHPC mixture consisted of Type I/II portland cement, silica fume, fly ash, HRWRA, water, and 1.5\% steel fibers by volume. The sand, cement, and fly ash were obtained from local sources, and the silica fume, steel fibers, and HRWRA were obtained from regional suppliers.

\subsection{Bonded specimens}

Specimens were produced by casting the substrate, moist curing the substrate to an age of seven days, casting the UHPC portion of the specimen, and then moist curing for another seven days. The composite specimens were then tested for seven and 28 day strengths (measured from the time that the UHPC portion of the specimen was cast).

Table 1. Non-proprietary UHPC mixture proportions.

\begin{tabular}{|c|c|c|}
\hline Materials & $\mathbf{K g} / \mathbf{m}^{\mathbf{3}}$ & $\left(\mathbf{l b} / \mathbf{y d} \mathbf{d}^{\mathbf{3}}\right)$ \\
\hline Cement & 817 & $(1377)$ \\
\hline Silica Fume & 102 & $(172)$ \\
\hline Fly Ash & 102 & $(172)$ \\
\hline Sand & 1009 & $(1702)$ \\
\hline HRWRA L/m $\mathbf{3}\left(\mathbf{g a l} / \mathbf{y d}^{\mathbf{3}}\right)$ & 45 & $(9.09)$ \\
\hline Water & 153 & $(258)$ \\
\hline Steel Fibers & 119 & $(201)$ \\
\hline
\end{tabular}

Table 2. Substrate concrete mixture proportions.

\begin{tabular}{|c|c|c|}
\hline Materials & $\mathrm{Kg} / \mathrm{m}^{3}$ & $\left(\mathrm{lb} / \mathrm{yd}^{3}\right)$ \\
\hline Cement & 344 & $(580)$ \\
\hline Coarse Aggregate & 98 & $(1660)$ \\
\hline Sand & 719 & (1212) \\
\hline Water & 172 & $(290)$ \\
\hline $\begin{array}{c}\text { Air-Entraining Admixture } \\
\mathbf{L} / \mathbf{m}^{3}\left(\text { fl.oz. } / \mathbf{y d}^{3}\right)\end{array}$ & 1.22 & (29) \\
\hline
\end{tabular}

\subsection{Bond strength testing}

Two different tests are being performed to assess the bond strength between the substrate and the UHPC grout. These tests are the slant shear and direct tension tests. These tests are being performed on composite specimens with substrate textures that included a formed surface and chipped textures with depths of $0.100 \mathrm{~mm}$ (0.004 in), $0.128 \mathrm{~mm}(0.00503 \mathrm{in})$, and $1.126 \mathrm{~mm}$ (0.0496 in). Texture depth was determined in accordance with ASTM E965. Surface preparation varies from formed to medium roughness. The formed surface was prepared by using a stiff wire brush to dust off loose material and the chipped surfaces were prepared by using an air hammer to chip off the formed surface. Figure 1 illustrates the two substrate textures.

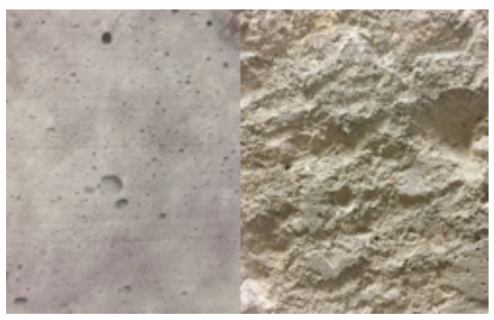

Fig. 1. Substrate textures: formed (left) and chipped (right).

\subsection{Slant-shear testing}

The slant-shear strength of the composite bonded specimens was determined in accordance with ASTM C882. Normal strength concrete was cast in a 152 by 304 $\mathrm{mm}$ ( 6 by 12 in) cylindrical mold. The mold uses a plastic dummy insert to produce a $60^{\circ}$ incline. The specimens are moist cured for seven days prior to texturing (if needed) and placement of UHPC. After demolding, the composite specimens are cured at ambient conditions $\left(20^{\circ} \mathrm{C}\left[68^{\circ} \mathrm{F}\right]\right.$ and $30 \%$ relative humidity). After seven more days of curing, the specimens are tested in compression to determine the shear strength of the bond.

The slant-shear specimens were tested in compression, as shown in Figure 2, until failure. Three possible failure modes can occur that include failure in the UHPC, failure of the bond, and failure in the substrate. Shear strength of the bond was calculated using the following equation:

$$
\tau=(\mathrm{P} / \mathrm{A}) \sin (\alpha) \cos (\alpha)
$$

where $\tau=$ shear stress, $\mathrm{P}=$ ultimate load, $\mathrm{A}=$ crosssectional area, and $\alpha=$ angle of the bonded interface from horizontal $\left(60^{\circ}\right)$.

\subsection{Direct tension testing}

Direct tensile strength of bonded specimens is being evaluated using a modified version of ASTM C1583. The standard test uses a pull-off device to determine the strength from cored slabs. For this research, that device was replaced with the tension test apparatus shown in Figure 3 to test cylindrical cores taken from prismatic specimens.

\subsection{Scanning electron microscopy}

Scanning electron microscopy was performed to provide a detailed characterization of the fractured surfaces. 
Images were collected for both formed and chipped surfaces to provide a visual explanation of the microstructure of each fracture specimen.

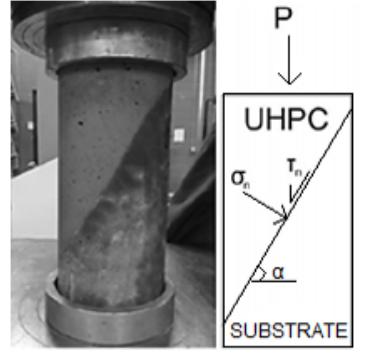

Fig. 2. Test setup for the slant shear specimen.

\section{Results and discussion}

\subsection{Slant-shear strength}

Table 3 presents the results obtained from slant-shear testing. According to ACI 546 [14], the recommended minimum slant-shear strength is $7 \mathrm{MPa}(1000 \mathrm{psi})$ at seven days and $14 \mathrm{MPa}$ (2000 psi) at 28 days. Table 3 shows that all of the strengths were adequate at seven days and that the average bond strengths were adequate at 28 days. It is important to note that ACI 546 [14] recommends a minimum texture depth of $6.25 \mathrm{~mm}(0.25$ in), which is much greater than the depth of even the deepest chipped textures studied in this work. However, it is difficult to produce adequate texture in the field during shear key repairs, so the texture depths investigated in this work are probably representative of what should be expected in the field.

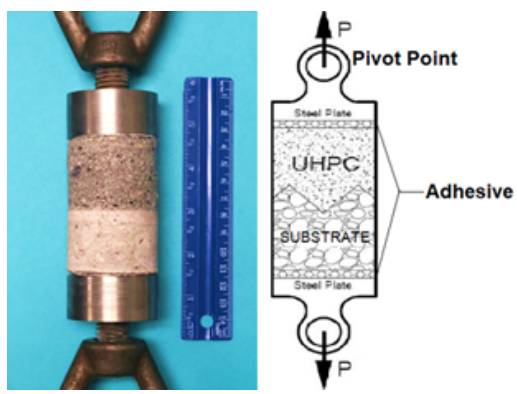

Fig. 3. Test setup for the direct tension test.

\subsection{Direct tensile strength}

Table 4 presents the results obtained from the direction tension tests. According to ACI 546 [14], the recommended bond strength from direct tension tests should be $1 \mathrm{MPa}(150 \mathrm{psi})$. Table 4 shows that the average strengths for both textures meet the ACI recommendation. Again, adequate bond strength was achieved even with inadequate texture.
Table 3. Slant shear test results.

\begin{tabular}{|c|c|c|c|c|}
\hline \multirow{2}{*}{ Texture } & \multicolumn{2}{|c|}{ 7 Day Strength } & \multicolumn{2}{c|}{ 28-Day Strength } \\
\cline { 2 - 5 } & MPa & psi & MPa & Psi \\
\hline \multirow{4}{*}{ Formed } & 22.8 & 3300 & 18.5 & 2680 \\
\cline { 2 - 5 } & 9.7 & 1400 & 19.8 & 2870 \\
\cline { 2 - 5 } & 20.0 & 2900 & 11.7 & 1700 \\
\cline { 2 - 5 } & 28.7 & 4160 & -- & -- \\
\hline \multirow{2}{*}{$\begin{array}{c}\text { Chipped } \\
\text { 0.221 mm (0.008 in) }\end{array}$} & 29.4 & 4730 & 33.7 & 4890 \\
\cline { 2 - 5 } & 23.8 & 3450 & 29.9 & 4340 \\
\cline { 2 - 5 } Chipped & 35.9 & 5200 & 35.9 & 5200 \\
\hline \multirow{3}{*}{ C.701 mm (0.0276 in) } & 28.7 & 4160 & 35.4 & 5130 \\
\hline \multirow{2}{*}{ Chipped } & 25.8 & 3740 & 32.3 & 4690 \\
\cline { 2 - 5 } $\mathbf{1 . 2 6}$ mm (0.0496 in) & 29.4 & 4270 & 25.8 & 3740 \\
\cline { 2 - 5 } & 15.7 & 2270 & 22.1 & 3210 \\
\hline \multirow{2}{*}{ mm.1 } & 15.190 & 27.4 & 3980 \\
\hline
\end{tabular}

\subsection{Scanning electron microscopy}

A sample of the scanning electron microscopy images obtained in this work are presented in Figure 4. In the left image, the arrow indicates fly ash residue that remained bonded to the substrate surface after fracture. This provides an indication that the supplementary cementitious materials are primarily responsible for the bond strength. The second image in Figure 4 shows steel fibers that are protruding from the UHPC after it debonded from the substrate. This observation could indicate that the steel fibers might obstruct some of the bond or that some of the fibers are not participating in the behavior of the UHPC.

Table 4. Direct tension test results.

\begin{tabular}{|c|c|c|c|c|}
\hline Texture & $7 \mathrm{Da}$ & rength & Averas & ength \\
\hline & MPa & psi & MPa & psi \\
\hline \multirow{8}{*}{ Formed } & 0.88 & 127 & \multirow{8}{*}{1.14} & \multirow{8}{*}{166} \\
\hline & 0.66 & 96 & & \\
\hline & 1.54 & 223 & & \\
\hline & 0.96 & 139 & & \\
\hline & 1.63 & 237 & & \\
\hline & 1.57 & 228 & & \\
\hline & 1.07 & 154 & & \\
\hline & 0.85 & 123 & & \\
\hline \multirow{2}{*}{$\begin{array}{c}\text { Chipped } \\
0.128 \mathrm{~mm} \\
(0.00503 \mathrm{in})\end{array}$} & 1.34 & 194 & \multirow[b]{2}{*}{1.17} & \multirow[b]{2}{*}{170} \\
\hline & 1.00 & 145 & & \\
\hline
\end{tabular}

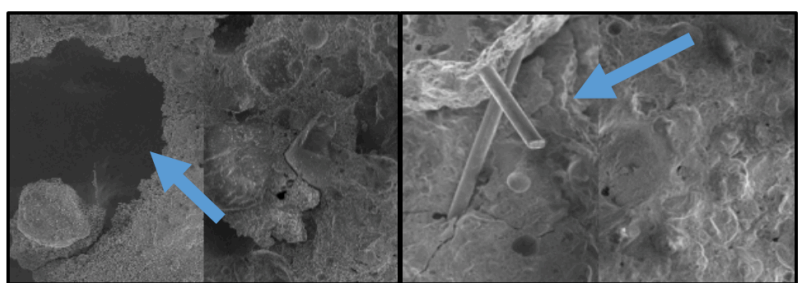

Fig. 4. Formed UHPC and normal concrete (left) and texturize UHPC and normal concrete (right).

\section{Ongoing work}

Ongoing work associated with this project includes: 
1. Assessment of early-age and longer-term shrinkage of the UHPC mixture since shrinkage of the UHPC grout can cause both shear and tensile stresses on the bond between the grout and a girder.

2. Additional scanning electron microscopy imaging to study the quality of the bond between the UHPC grout and various substrate surfaces.

3. Full-scale structural testing of UHPC shear keys between adjacent pre-stressed channel girders.

\section{Conclusions}

The following conclusions have been drawn from this work:

1. Adequate bond strength can be achieved between UHPC and substrate concrete with the proper surface preparation, even when texture depth is inadequate.

2. Quality of the bond seems to be largely dependent upon supplementary cementitious material particles penetrating into the substrate concrete.

The authors would like to thank Tran-SET for funding the project, the New Mexico Department of Transportation for their continued support of UHPC research and collaboration, and BASF Chemical Company for their donation of silica fume and admixtures.

\section{References}

1. Hussein, H., Sargand, S., and Steinberg, E. (2018). Shape Optimization of UHPC Shear Keys for Precast, Prestressed, Adjacent Box-Girder Bridges. J. Bridge Eng., 23(4).

2. Weldon, B.D., Jauregui, D.V., Newtson, C.M., Taylor, C.W., Montoya, K.F., Allena, S., Muro, J., Tahat, M., Lyell, E., and Visage, E.T. (2012). Feasibility Analysis of Ultra-High Performance Concrete for Prestressed Concrete Bridge Applications, Report NM09MCS-01, New Mexico Department of Transportation.

3. Newtson, C.M., Weldon, B.D., Al-Basha, A.J, Manning, M.P., Toledo, W.K., and Davila, L.D. (2018). Bridge Deck Overlays Using Ultra-High Performance Concrete. Tran-SET final research report for project 17CNMS01, Transportation Consortium of South-Central States (Tran-SET).

4. Ozyildirim, C., and Moruza, G. (2016). HighPerformance Grouting Materials in Shear Keys Between Box Beams. Transportation Research Record, Journal of the Transportation Research Board, 2577, 35-42.

5. Montoya, K. (2010). Feasibility of Using Ultra High Performance Concrete in New Mexico Bridge Girders, M.S. thesis, New Mexico State University.
6. Haber, Z.B., Graybeal, B.A., and Munoz, J.F. (2017). Field Testing of an Ultra-High Performance Concrete Overlay. FHWA-HRT-17-096, Federal Highway Administration.

7. Magureanu, C., Sosa, I., Negrutiu, C., and Heghes, B. (2012). Mechanical Properties and Durability of Ultra-High Performance Concrete. ACI Materials Journal, 109(2), 177-183.

8. Naaman, A.E. and Wille, K. (2012). The path of Ultra-High performance Fibre Reinforced Concrete (UHP-FRC): Five Decades of Progress. Third International Symposium on UHPC and Nanotechnology for High Performance Construction Materials, Kassel, 3-15.

9. Shann, S.V., Harris, D.K., Carbonell, M.C., and Ahlborn, T.M. (2012). Application of UHPC as a Thin Topped Overlay for Concrete Bridge Decks. Third International Symposium on UHPC and Nanotechnology for High Performance Construction Materials, 929-936.

10. Yuan, J., Graybeal, B. (2016). Full-Scale Testing of Shear Key Details for Precast Concrete Box-Beam Bridges. J. Bridge Eng., 21(9).

11. Miller, R.A., Hlavacs, G.M., Long, T., and Greuel, A. (1999). Full-Scale Testing of Shear keys for Adjacent Box Girder Bridges. PCI Journal, 44(6), 80-90.

12. Grace, B.F., Jensen, E.A., Bebawy, M.R. (2012). Transverse post-tensioning arrangement for side-byside box-beam bridges. PCI Journal, 57(2), 48-63.

13. Hanna, K.E., Morocous, G., Tadros, M.K. (2009). Transverse Post-Tensioning Design and Detailing of Precast, Prestressed Concrete Adjacent-Box-Girder Bridges. PCI Journal, 56(4), 160-174.

14. ACI Committee 546R-04, (2004). Guide to Materials Selection for Concrete Repair, American Concrete Institute ACI546R-04. 